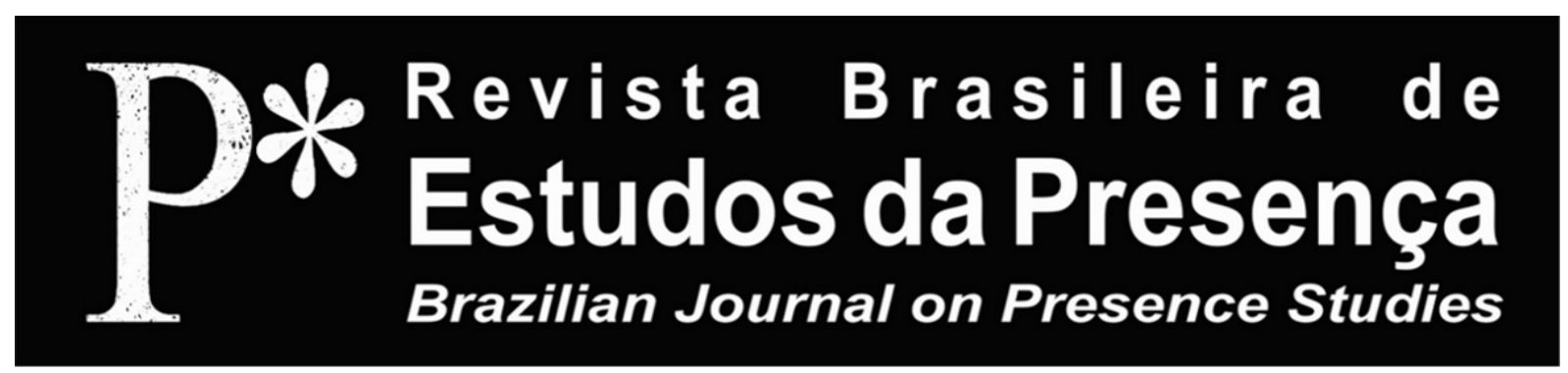

E-ISSN 2237-2660

\title{
L'Expérience Esthétique, une Nouvelle Conquête Démocratique
}

\author{
Alain Kerlan \\ Université Lumière Lyon 2 - Lyon, France
}

RÉSUMÉ - L'Expérience Esthétique, une Nouvelle Conquête Démocratique - Ce texte a pour point de départ les Lettres sur l'Éducation Esthétique de l'Homme du poète dramaturge et philosophe Friedrich Von Schiller. Il commence par en montrer la grande actualité politique et éducative, et insiste sur la nécessité d'en relancer aujourd'hui le geste, dans la perspective d'une nouvelle et nécessaire étape de la démocratisation culturelle. Se référant ensuite à l'esthétique de John Dewey, et prenant appui sur les travaux contemporains consacrés aux artistes engagés dans la tâche éducative, il interroge les intérêts et les interrogations que l'art et l'éducation ont aujourd'hui en commun.

Mots-clés: Expérience Esthétique. Démocratie. Éducation Esthétique. Subjectivation. Normativité.

ABSTRACT - Aesthetic Experience, a New Democratic Conquest - This paper has for starting point the Letters on the Aesthetic Education of Man by the poet, playwright, and philosopher Friedrich Von Schiller. It begins by showing how politically and educationally current this text is, and insists on the necessity of boosting this gesture today, with the prospect of a necessary new stage of the cultural democratization. Referring then to the aesthetics of John Dewey, and taking support on the contemporary works dedicated to the artists committed in the educational task, it explores the interests and the questionings that the art and the education have in common today.

Keywords: Aesthetic Experience. Democracy. Aesthetic Education. Subjectivity. Normativity.

RESUMO - A Experiência Estética, uma Nova Conquista Democrática - Este artigo parte das Cartas sobre a Educação Estética do Homem, do poeta, dramaturgo e filósofo Friedrich Von Schiller. Ele começa mostrando a atualidade política e educativa desse texto e insiste sobre a necessidade de retomar o gesto hoje na perspectiva de uma etapa nova e necessária para a democratizaçáo cultural. Ao fazer referência também à estética de John Dewey e apoiando-se em trabalhos contemporâneos sobre artistas envolvidos na tarefa educacional, este artigo problematiza os interesses e as questóes que a arte e a educaçáo têm em comum hoje em dia.

Palavras-chave: Experiência Estética. Democracia. Educação Estética. Subjetivação. Normatividade. 
Il fut un temps où des princes éclairés pouvaient prêter une oreille attentive aux propos des philosophes; même lorsque ces propos touchaient aux choses de l'éducation. C'est ainsi qu'en 1795, le poète, dramaturge et philosophe Friedrich Von Schiller adressait au Duc Chrétien-Frédéric de Holstein-Augustenbourg ses fameuses Lettres sur l'Éducation Esthétique de l'Homme: un plaidoyer sans précédent pour une politique qui fasse résolument de l'éducation esthétique la base de l'éducation. Ces temps sont-ils définitivement révolus? On voudrait bien ne pas le croire, et espérer qu'une cause indissolublement éducative et politique puisse encore être entendue, et puisse bénéficier des éclairages et des débats publics plus que jamais nécessaires ${ }^{1}$. Ni le propos développé ici ni son auteur n'ont l'ambition et la prétention d'élever le débat à la hauteur où l'avait établi Schiller: ils s'efforcent simplement d'en saisir la portée de nos jours encore, d'éclairer la signification et l'importance - pour la démocratie et l'éducation nécessaire à la démocratie - du lien qu'établit Schiller entre l'esthétique et la liberté.

\section{Les Trois Âges de la Démocratisation}

Considérer l'expérience esthétique comme un enjeu démocratique peut surprendre, d'autant plus que les difficultés que connait aujourd'hui la démocratie et les crises qu'elle traverse, comme les menaces de tous ordres qui pèsent sur elle et sur ses valeurs en bien des points du globe, semblent reléguer à l'arrière-plan des préoccupations légitimes ces affaires d'art et d'esthétique. C'est pourtant bien de cela qu'il s'agit, et que le propos inaugural de Schiller nous aide à comprendre: ce qui se joue au sein de l'éducation artistique, la conjonction sans équivalent du faire, du sentir et du penser, l'éducation de ce que l'on peut appeler, avec le philosophe Jean-Marie Schaeffer (2000) la conduite esthétique, participe pleinement d'une authentique éducation démocratique. L'enjeu de l'éducation artistique est bel et bien un enjeu pleinement politique. Et il l'est plus que jamais à l'ère numérique.

La thèse que voudrait avancer et illustrer cette contribution peut s'énoncer de façon assez simple, dans le langage de la politique culturelle: elle invite à considérer que nous sommes engagés dans une troisième étape, une troisième phase ou une troisième strate de ce qu'il est convenu d'appeler la démocratisation culturelle. La 
première phase consistait et consiste toujours à permettre à tous et à chacun d'accéder au patrimoine de l'art et de la culture, à accorder à chacun de s'approprier le patrimoine qui lui appartient en droit en tant qu'homme/femme et citoyen. Inutile de rappeler la nécessité démocratique de cette appropriation. Nous savons bien qu'elle est loin d'être accomplie, et c'est pourquoi il est préférable de parler de strate, plutôt que de phase: une strate demeure active. La seconde strate, donc, est caractérisée par l'ambition de permettre à chacun d'accéder aux pratiques artistiques de son choix. Il s'agit là encore, chacun en conviendra, d'une autre dimension, tout aussi nécessaire, de la démocratisation. Et dans ce domaine aussi bien des efforts de réalisation, parfois même de conviction, doivent encore être consentis. Pour quelques classes de collège bénéficiant du dispositif orchestre à l'école - né en Amérique du sud, et connaissant un succès croissant en Europe - certes de plus en plus nombreuses et pour lesquelles l'école ouvre les portes du théâtre, combien d'élèves ignoreront pour toujours la pratique instrumentale, le jeu dramatique?

Y remédier, est-ce pour autant suffisant? Eh bien non. Il existe une autre dimension, que nous découvrons aujourd'hui comme une nouvelle exigence démocratique, une dimension et une ambition, qu'on peut qualifier de fondatrice ou de refondatrice: permettre à tous et à chacun d'accéder à une véritable expérience esthétique. Une nouvelle et essentielle étape de la démocratisation dans le domaine de l'art et de la culture passe par l'accès de tous à l'expérience esthétique, comme expérience humaine fondamentale.

Encore faut-il expliquer et comprendre pourquoi. On se propose ici d'apporter au moins quelques éclairages. Les premiers empruntent directement à la pensée philosophique dans son histoire: la portée politique de l'éducation artistique, en effet, et pour m'en tenir à une période qui correspond à l'histoire moderne de la démocratie, a été déjà pleinement pensée dès la fin du XVIII ${ }^{e}$ siècle. Les seconds éclairages procèdent d'une interrogation plus directe. Constatant que l'éducation et les éducateurs d'aujourd'hui se tournent de plus en plus volontiers vers l'art, et que l'art et les artistes de leur côté manifestent par leur engagement l'intérêt porté au champ éducatif, il convient d'interroger cette convergence, cette configuration: qu'est-ce qui rapproche aujourd'hui l'art et l'éducation? Quels objectifs, quelles interrogations ont-ils en commun, en partage? 


\section{«C'est par la Beauté qu'on s'Achemine vers la Liberté»}

La première série d'éclairages commence par une lecture. Lecture du courrier, si l'on peut dire, puisqu'il s'agit de quelques extraits de ces Lettres rédigées par Friedrich Von Schiller, à la lumière desquelles s'est engagée notre réflexion. Pour en apprécier toute la portée, il faut tout de même préciser qui en était exactement le destinataire, ce duc Chrétien-Frédéric de Holstein-Augustenbourg. Un inconnu pour nous tous, sans doute, aujourd'hui, mais alors un puissant, rien moins qu'un prince éclairé détenteur de la puissance politique. Et c'est bien à celui dont peut dépendre le sort politique d'un peuple que le poète s'adresse. Le début de sa toute première lettre donne le ton:

Vous voulez donc bien m'accorder la faveur de vous présenter dans une série de Lettres les résultats de mes investigations sur la Beauté et sur l'Art. Je sens vivement le poids de mon entreprise, mais aussi son attrait et sa dignité. Le sujet dont je vais parler a un rapport immédiat avec notre bonheur, avec ce qu'il y a de meilleur en lui, et il a un rapport assez étroit avec la noblesse morale de la nature humaine ${ }^{2}$ (Schiller, 1992, p. 81).

La hauteur à laquelle le poète situe l'Art et la Beauté sur l'échelle des valeurs ne laisse aucun doute: la plus élevée, puisqu' il s'agit autant du bonheur que de la noblesse morale de l'humanité, et mieux encore de leur conjonction: dans l'art et la beauté, bonheur et moralité, bonheur et vertu marchent ensemble. Utopie? Non, si utopie signifie chimère, mais au moins horizon vers lequel marcher. Schiller n’a rien d'un naïf qui s'enflamme et ignore tout de l'état du monde. L'extrait de la deuxième lettre montre bien qu'il ne méconnait en rien la difficulté à se faire entendre que ne manquera pas de rencontrer son plaidoyer politique pour l'art et l'esthétique:

Maintenant, c'est le besoin qui règne en maître et qui courbe l'humanité déchue sous son joug tyrannique. Lutilité est la grande idole de l'époque; elle demande que toutes les forces lui soient asservies et que tous les talents lui rendent hommage. Sur cette balance grossière le mérite spirituel de l'art est sans poids; privé de tout encouragement, celui-ci se retire de la kermesse bruyante du siècle (Schiller, 1992, p. 89).

Maintenant? Le maintenant de Schiller date, rappelons-le, de l'année 1795. Qui pourrait nier qu'il concerne plus que jamais notre présent, en ce début du XXI ${ }^{e}$ siècle mondialisé, où plus que jamais le besoin «[...] règne en maître» et impose son joug, où plus que jamais 
«[...] l'utilité est la grande idole de l'époque»? Où «l'utilité» dont se réclament les forces économiques mondialisées s'acharnent à asservir les autres forces, et même celles de l'art et de la culture, sommées de s'en remettre aux exigences des industries du divertissement? Oui, que pèse alors le «mérite spirituel» de l'art qui y résiste, que pèse dans cette «balance grossière» la simple valeur esthétique que le poète prétend porter au plus haut? La réponse se trouve à la fin de cette même deuxième lettre; elle délivre le message capital que Schiller juge nécessaire d'adresser au politique:

J'espère vous persuader que cette matière est beaucoup plus étrangère au goût du siècle qu'à ses besoins, et que même pour résoudre dans l'expérience le problème politique dont j'ai parlé, la voie à suivre est de considérer d'abord le problème esthétique; car c'est par la beauté que l'on s'achemine à la liberté. C'est là une démonstration qui ne peut pas être menée sans que je vous rappelle les principes que d'une manière générale la Raison prend pour guides quand elle édicte une législation politique (Schiller, 1992, p. 91).

On n'entrera pas ici dans la démonstration que développait, à l'intention du Duc, le dramaturge philosophe, encore tout imprégné de la lecture de cette Critique du jugement alors toute récente et dans laquelle Emmanuel Kant donnait au sentiment esthétique sa pleine signification anthropologique. On se contentera de souligner ce que tout lecteur attentif aura assurément noté: oui, Schiller nous dit bien, et juge nécessaire, de faire savoir à la puissance politique que pour résoudre le problème politique, c'est-à-dire le problème de la liberté, c'est-à-dire le problème même de la démocratie, le chemin passe par l'esthétique. Oui, quelque chose d'essentiel pour la liberté est en jeu dans ce rapport sensible très particulier que nous entretenons avec les œuvres d'art mais aussi avec les œuvres de la nature, et bien d'autres objets ou situations, et qu'expriment le plaisir esthétique, le sentiment esthétique, le geste esthétique, le jugement esthétique. Quelque chose d'essentiel et d'une certaine façon quelque chose de préalable: la «beauté», nous dit Schiller dans le langage de l'esthétique de son temps, précède d'une certaine façon la liberté; pas la liberté comme principe, celle-ci est toujours déjà là, c'est la leçon philosophique du XVIII siècle, siècle des révolutions, mais la liberté dans son effectivité, dans son accomplissement.

La conséquence pour nous encore capitale qu'en tire Schiller et qu'il adresse au Duc est formulée dans de nombreux autres extraits des 
Lettres, dont on ne saurait trop recommander la lecture. Contentonsnous ici de la résumer. Puisque vous daignez m'entendre, lui dit-il en substance, vous qui avez le pouvoir de favoriser le règne effectif de la liberté parmi les hommes, vous devez commencer par former les hommes en sorte qu'ils soient à même de rendre cette liberté effective; et pour cela une voie est nécessaire: il faut commencer par l'éducation esthétique. Pour le dire dans notre langage contemporain: l'éducation à la citoyenneté passe par l'éducation esthétique, quelque chose de l'éducation à/pour la démocratie y est engagé.

Les Lettres parurent pour la première fois dans le journal Les Heures, en 1795. Elles furent réimprimées en 1801, et l'édition se fait sous ce titre: Lettres sur l'Éducation Esthétique de l'Homme (Über die Ästhetische Erziehung des Menschen). Dans Les Heures, la première lettre était précédée de cette épigraphe française de Jean-Jacques Rousseau: si c'est la raison qui fait l'homme, c'est le sentiment qui le conduit. Pour la première fois, l'éducation esthétique était pensée dans une perspective politique globale. Schiller partait de la Révolution française, selon lui inaccomplie. Parce que l'humanité est divisée entre raison et instinct et que ces deux dimensions de l'homme total s'affrontent, pensait-il, la liberté ne peut s'épanouir. Le plaisir esthétique seul peut réconcilier l'esprit et les sens, et donner naissance à une société harmonieuse, équilibrée, juste, accomplie. Les artistes à ses yeux sont les meilleurs artisans du progrès politique, comme du progrès tout court. Une précision essentielle: l'éducation esthétique n'est pas ici un complément éducatif, qui viendrait après les autres: il s'agit bien de dire - c'est le sens fort du titre, Lettres sur l'Éducation Esthétique de l'Homme - que seule l'éducation esthétique éduque pleinement, totalement.

\section{La Critique Artiste en Éducation: brève généalogie}

La thèse révolutionnaire de Schiller n'est donc pas une pensée isolée et sans lendemains. Elle a connu un écho peut-être souterrain mais assurément considérable, même si au bout du compte il est peu étudié. Sans lui donner tout l'arrière-plan des thèses philosophiques concernant les relations de l'art et de la politique qu'il serait nécessaire d'évoquer pour bien la situer, tâche que son ampleur interdit ici, et en s'en tenant au thème de l'éducation esthétique comme condition de la liberté politique qui en est le trait central, il est possible d'y voir 
l'un des tout premiers maillons généalogiques de ce que nous appellerons, en nous inspirant de Luc Boltanski et Eve Chiapello (1999), la «critique artiste» en éducation.

Cette généalogie trouverait son impulsion dans l'œuvre politique et éducative de Jean-Jacques Rousseau. L'œuvre de Rousseau alterne et mêle à la fois les deux critiques que distingue Boltanski au début de son ouvrage Le nouvel esprit du capitalisme (1999): la "critique sociale» et la "critique artiste». Toutefois, du Discours sur l'Origine de l'Inégalité au Rêveries (Rousseau, 1959), en passant par l'Emile (Rousseau, 1969), on peut lire une évolution générale de l'œuvre telle que la critique artiste semble bien supplanter ou au moins rattraper la critique sociale.

Trouverait place également dans notre généalogie le mouvement saint-simonien, et plus précisément ce qui se jouait au sein du petit groupe d'ouvriers saint-simoniens qui, autour de 1830, voulut croire que la seule émancipation véritable, la seule voie pour s'arracher à la contrainte de l'existence prolétarienne, passait par l'art, par l'écriture, la poésie. Jacques Rancière leur a consacré une magistrale étude dans ce magnifique ouvrage intitulé La Nuit des Prolétaires (1981), et non moins magnifiquement sous-titré Archives du Rêve Ouvrier.

Il existe aussi une pensée socialiste particulièrement sensible à la portée éducative de l'art et de l'esthétique. La pensée de William Morris et son ouvrage de 1884, Art et Socialisme, en sont des formulations qu'une généalogie de la critique artiste en éducation se devrait de prendre en compte. D’une façon générale, un projet généalogique devrait s'attacher à ces pensées où s'articulent socialisme et romantisme. Dans le sillage de William Morris, il trouverait un ouvrage qui exerça une forte influence, jusqu'en Amérique du Sud, le Education through Art de Herbert Read, publié en 1943. Le propos qu'y tenait Read fait fortement écho à celui de Schiller, comme l'illustre ce passage de The Education of Free Men: "We are made perfect by natural habits, but slaves by social conventions; and until we have become accustomed to beauty we are not capable of truth and goodness, for by beauty we mean the principle of harmony which is the given order of the physical universe, to which we conform and live, or which we reject and die» (Read, 1944, p. 25). L'entremêlement de la critique sociale et de la critique artiste est ici patent. Comme il l'est chez Herbert Marcuse, dont l'écho schillérien peut s'entendre 
dans le titre même de son dernier ouvrage, The Aesthetic Dimension (1978). Comme le laisse entendre son sous-titre, Toward a Critique of Marxist Aesthetics, Marcuse y engageait une critique de la critique sociale, à ses yeux inachevée tant qu'elle était incapable d'intégrer la dimension émancipatrice de l'esthétique.

Pour l'époque contemporaine, notre brève esquisse généalogique ne peut malheureusement qu'évoquer deux auteurs d'inspirations différentes où s'exprime néanmoins la préoccupation politique de l'esthétique, y compris dans sa dimension éducative. Dans Le Partage du Sensible (2000), le premier, Jacques Rancière, développe, dans une relation critique à la pensée marxiste, une conception du politique comme partage $d u$ sensible que l'on peut lire dans le sillage de son œuvre initiale consacrée aux ouvriers saint-simoniens, et qui déplace et relance la thèse de Schiller. Le second auteur, Joëlle Zask, dans Art et Démocratie: les peuples de l'art (2003), puise à une toute autre source, le pragmatisme de John Dewey, sur lequel nous allons nous arrêter plus longuement. Au préalable, et pour donner un horizon à notre esquisse généalogique, notons qu'il y aurait lieu de s'interroger sur l'influence que Schiller exerça en France sur un André Malraux, sur un Jacques Lang, tous deux ministres de la culture en des temps différents, et dont l'action selon des voies certes elles-mêmes différentes aura porté l'exigence d'art et de culture comme une exigence politique. Une histoire mondiale de la réception et de l'influence des Lettres sur l'Éducation Esthétique de l'Homme mériterait d'être mise en chantier.

\section{L'Esthétique et l'Homme Ordinaire}

Le second éclairage philosophique sur lequel nous nous arrêtons occupe une place à part dans notre généalogie. C'est celui du philosophe américain John Dewey, fondateur de ce pragmatisme si caractéristique de la sphère anglo-saxonne, l'équivalent pour la philosophie américaine et anglophone de ce qu'est en France le cartésianisme. Nous quittons donc la fin du XVIII siècle et le tout début du XIX ${ }^{\mathrm{e}}$ siècle, pour aborder le $\mathrm{XX}^{\mathrm{e}}$ siècle. Mais nous ne quittons pas ce carrefour de l'esthétique et de la démocratie qu'on tente ici d'éclairer. John Dewey est sans doute bien connu comme penseur de la démocratie, auteur de Democracy and Education: an introduction to the philosophy of education, ouvrage publié en 1916, et dont l'influence a été consi- 
dérable; mais il est aussi l'auteur de Art as Experience (2005), paru en 1934, et dont on mesure peut-être mieux aujourd'hui l'importance.

Pour résumer brièvement - mais sans doute de façon un peu trop abrupte - l'apport de John Dewey à notre sujet, il faut dire qu' il nous permet de désenclaver l'expérience esthétique de son carcan exclusivement culturel et patrimonial, pour la restituer à l'homme ordinaire, à l'expérience ordinaire Quelques mots donc sur ce désenclavement de l'expérience esthétique. Les quelques lignes que nous empruntons à L'Art comme Expérience feront clairement comprendre de quoi il s'agit:

Afin de comprendre l'esthétique dans ses formes accomplies et reconnues, on doit commencer par la chercher dans la matière brute de l'expérience, dans les événements et les scènes qui captent l'attention auditive et visuelle de l'homme, suscitent son intérêt et lui procurent du plaisir lorsqu'il observe et écoute, tels les spectacles qui fascinent les foules: la voiture des pompiers passant à toute allure, les machines creusant d'énormes trous dans la terre, la silhouette d'un homme, aussi minuscule qu'une mouche, escaladant la flèche du clocher, les hommes perchés dans les airs sur des poutrelles, lançant et rattrapant des tiges de métal incandescent. Les sources de l'art dans l'expérience humaine seront connues de celui qui perçoit comment la grâce alerte du joueur de ballon gagne la foule des spectateurs, qui remarque le plaisir que ressent la ménagère en s'occupant de ses plantes, la concentration dont fait preuve son mari en entretenant le carré de gazon devant la maison, l'enthousiasme avec lequel l'homme assis auprès du feu tisonne le bois qui brûle dans l'âtre et regarde les flammes qui s 'élancent et les morceaux de charbon qui se désagrègent (Dewey, 2005, p. 23).

La citation est sans doute un peu longue, mais il est nécessaire de lire ce passage jusqu'au bout des exemples qu'il enchaîne. Sa portée en effet tient au contenu de chaque situation évoquée, et à leur enchaînement. Le lecteur aura peut-être vu passer devant ses yeux, à l'écoute de ce propos, le souvenir de telle ou telle ouvre d'art célèbre, par exemple une photographie de Doisneau, de Boubat, ou de Willy Ronis, où des ouvriers perchés sur des poutrelles dessinent leur frêles silhouettes sur le vide du ciel; il aura peut-être évoqué le souvenir de telle ou telle ouvre musicale intégrant les bruits de la rue, comme une composition d'Edgar Varèse. Il ne s'agit pourtant que d'expériences ordinaires, et même empreintes d'une jubilation toute enfantine; il s'agit de l'expérience de tous et de chacun, la 
mienne, la vôtre, sans doute. Même le jardinage, l'entretien patient du gazon, le simple plaisir de la rêverie devant le feu que célébrera Bachelard, sous un certain angle, méritent selon Dewey d'être élevés à la dignité esthétique; et le football lui-même, à certains égards. Dewey procède ainsi à une radicale démocratisation de l'expérience esthétique. Certes, elle est à son apogée dans ses formes accomplies et reconnues que sont les œuvres que conservent les musées ou que donnent à voir et à entendre les salles de théâtre et de concerts; mais elle est déjà là, tout entière, dans toute expérience véritable, dans toute expérience authentique. Elle est à son sommet dans les toiles qu'inspire à Nicolas de Staël le spectacle du football; mais elle est déjà là en puissance dans ce spectacle même. Elle n'appartient pas exclusivement dans son essence à l'exception de la culture seconde, recueillie dans l'écrin muséal, mais elle est déjà là dans la culture première. Cette distinction culture première/culture seconde est empruntée à un grand intellectuel québécois, Fernand Dumont (1968), lequel voyait, dans la culture seconde, à la manière de John Dewey, une reprise de la culture première. Et en effet, osons le demander: à quoi bon le musée si le tableau n'est pas attendu et lu comme cristallisation d'une expérience du monde qui recoupe la mienne? À quoi bon la musique, la danse et la chorégraphie si le mouvement des danseurs sur la scène et le jeu des instruments ne prolongent d'une certaine façon ceux de mon propre corps dans l'expérience ordinaire? À quoi bon le théâtre si la scène où jouent les acteurs ne fait pas converger en une expérience partagée les scènes intérieures que portent en eux les spectateurs? Sous l'œuvre, quelle qu'elle soit, il y a en effet une relation au monde qui engage tout entier et doit aussi, et même d'abord, être éduquée.

On dira: la danse? Le théâtre? La peinture? Alors que l'avenir a pris son essor sur les ailes du numérique? Mais précisément: le défi éducatif du numérique et du virtuel rend plus que jamais nécessaire l'éducation esthétique. N'est-il pas capital que la relation sensible au monde précède la relation aux écrans? L'expérience esthétique partagée n'est-elle pas dès lors l'un des préalables éducatifs les plus essentiels? Cette éducation-là relie la culture et la sensibilité; elle fait de l'expérience esthétique le fondement de l'éducation artistique, et même l'une des bases de l'éducation. D'une éducation parmi les plus démocratiques qui soient. 
La philosophie esthétique et éducative de John Dewey donne du même coup à cette démocratisation de l'expérience esthétique - et à la démocratie elle-même - une dimension anthropologique, civilisationnelle. Elle fait de l'expérience esthétique une composante de base de notre humanité, de notre être-au-monde, l'un des vecteurs d'accomplissement de toute vie pleinement humaine. Voilà ce qu'écrit explicitement notre auteur:

L'expérience, lorsqu'elle atteint le degré auquel elle est véritablement expérience est une forme de vitalité plus intense. $\mathrm{Au}$ lieu de signifier l'enfermement dans nos propres sentiments et sensations, elle signifie un commerce actif et alerte avec le monde. A son plus haut degré, elle est synonyme d'interpénétration totale du soi avec le monde des objets et des événements (Dewey, 2005, p. 39).

Le programme éducatif que tire Dewey de cette philosophie esthétique n'est pas moins explicite: il s'agit, écrit-il, «[...] de restaurer cette continuité entre ces formes raffinées et plus intenses de l'expérience que sont les œuvres d'art et les événements quotidiens universellement reconnus comme des éléments constitutifs de l'expérience [...]», de «[...] rétablir la continuité entre l'expérience esthétique et les processus normaux de l'existence» $(2005$, p. 29).

\section{L'Entrée des Artistes}

Ce programme éducatif conçu voici près d'un siècle a conservé toute son actualité. C'est en effet l'enjeu de cette troisième phase de la démocratisation culturelle dans laquelle nous sommes engagés: rétablir la continuité entre l'expérience esthétique et les processus normaux de l'existence. Et c'est la tâche fondamentale à laquelle s'attellent de nos jours les artistes engagés dans le champ de l'éducation.

Venons-en donc à notre présent. Pour comprendre le sens du recours éducatif à l'art tel qu'il se développe aujourd'hui, il est nécessaire en effet de porter en tout premier lieu attention à un fait qui résume à lui seul la spécificité du mouvement social et culturel auquel ce recours est lié: l'appel aux artistes, l'entrée des artistes dans l'école. Si il y a quelque chose de nouveau et de déterminant dans le domaine de l'éducation artistique, depuis une bonne trentaine d'années, c'est bien la place qu'y prennent les artistes eux-mêmes. Donner à tout enfant, à tout jeune, la chance de vivre une authentique expérience esthétique, tel est le rôle dévolu aujourd'hui aux 
artistes dans l'école et l'éducation. L'appel aux artistes, l'entrée des artistes dans le champ éducatif, cet engagement des artistes en tant qu'artistes en éducation, participent d'une nouvelle étape dans/de la démocratisation artistique et culturelle. Ce processus, comme avait permis d'en prendre conscience le symposium international tenu à Paris au Centre Georges Pompidou en $2007^{3}$, n'est pas circonscrit à la seule Europe et au monde anglo-saxon: il tend à gagner de nombreux pays, du Nord au Sud, d'Ouest en Est, et le continent sud-américain n'y est pas étranger.

Le lecteur pourrait souhaiter à bon droit qu'on lui donne quelques exemples. Faute de pouvoir ici les présenter suffisamment, nous le renverrons aux Actes (2008) du symposium international consacré à l'éducation artistique tenu à Paris au Centre Georges Pompidou en janvier 2007, déjà signalés ci-dessus, où il en trouvera de nombreux, répartis dans de nombreux pays. Pour notre part, nous signalerons plus particulièrement deux des dispositifs français sur l'étude desquels s'appuie plus particulièrement notre propos. Le premier concerne la petite enfance. À Lyon, le Centre Enfance Art et Langages, créé et financé par la ville, permet d'accueillir en résidence de longue durée, sur une année entière au minimum et pour une durée hebdomadaire d'une dizaine d'heures, dans des écoles maternelles, des artistes de diverses disciplines, invités à travailler avec les enfants et les enseignants, à faire œuvre avec eux, à inscrire leur démarche artistique au cour des apprentissages, selon l'une des devises du Centre ${ }^{4}$. Le dispositif mis en place au collège Les Escholiers de la Mosson, à Montpellier, obéit au même principe. Les élèves d'une classe artistique expérimentale ont pu bénéficier, quatre années consécutives, tout au long du cycle des collèges, de compagnies d'artistes en résidence: compagnie théâtrale, compagnie chorégraphique, compagnie musicale. Elles aussi au cœur des apprentissages ${ }^{5}$.

Franchissons à présent un pas suplémentaire dans l'analyse de cette nouvelle étape de la démocratisation, en posant tout simplement les questions: pourquoi l'éducation se tourne-t-elle aujourd'hui du côté des artistes? Et pourquoi les artistes s'y engagent-ils? Pourquoi des artistes, pourquoi aujourd'hui? Posons donc explicitement la question: pourquoi des artistes, non seulement à la source de l'art ceci est la moindre des choses - mais à la source de l'éducation?

Voici la thèse que l'on défendra: si l'art et les artistes d'aujourd'hui sont impliqués et sollicités dans le champ éducatif, si même ils s’y 
engagent, c'est parce qu'il y a dans l'art d'aujourd'hui et la démarche artistique d'aujourd'hui, qu'elle se déploie sur le plan visuel, sonore, sur le plan émotionnel, ou sur ceux de l'énergie ou de la pensée, quelque chose qui touche aux questions et aux problèmes éducatifs majeurs, quelque chose qui touche à la source de l'éducation pour notre monde.

Ces questions, ces problèmes, gravitent autour de trois interrogations que l'art et l'éducation ont en partage: 1) une interrogation concernant l'individu, le sujet: comment éduquer, former le sujet dans ce qu'il est convenu d'appeler la société des individus, et dans laquelle précisément l'individu ne se veut plus seulement assujetti aux normes, mais source de normes? 2) interrogation sur la norme, donc: comment fabriquer de la norme et de la normativité, tout en invitant chaque sujet à être lui-même? 3) la question de la créativité, enfin, de la création: que recouvre la demande de création et de créativité qui concerne aujourd'hui chacun? Comment articuler créativité et normativité? Comment une norme peut-elle être immanente et néanmoins efficiente?

Ces trois points seront abordés succinctement ${ }^{6}$; sans doute il conviendrait de les situer et d'évoquer les différents travaux qui ont pu s'y consacrer; mais il ne s'agit ici que d'en faire saisir la portée politique.

\section{Former le Sujet}

Comment éduquer le sujet dans la société des individus? C'est assurément l'une des tâches les plus nécessaires et les plus difficiles à laquelle l'éducation et l'école sont aujourd'hui confrontées, à l'âge de l'individualisme démocratique. Et que peut apporter l'artiste à cette tâche? En quoi peut-il y contribuer?

Pour indiquer une piste de réflexion, arrêtons-nous sur une phrase qui ouvre le livre que le psychanalyste Daniel Sibony a consacré à l'art contemporain. Chaque artiste, écrit-il «[...] questionne l'art et le pratique d'une façon bien à lui, à la manière dont chaque homme est le seul à pouvoir vivre sa propre vie» (2005, p. 3). Insistons et soulignons. Etre en capacité de vivre sa propre vie comme seul soi-même on peut la vivre, n'est-ce pas l'expression la plus nette de l'individualisme contemporain, du souci de soi, et de ce qu'à la suite de Charles Taylor (2002) on appellera le souci de l'authenticité? Chacun a une 
manière unique de vivre sa propre vie; ou du moins s 'en donne le but. Si la tâche de chacun est de s'employer à l'accomplir, celle de l'éducateur n'est-elle pas de lui en donner l'élan et les moyens? Et dès lors l'artiste n'est-il pas par excellence l'éducateur qui convient?

Nous ne pouvons ici qu'indiquer brièvement ce que l'observation et l'analyse du travail des artistes en résidence en milieu scolaire permet d'avancer: la relation artiste/enfant (élève) est une relation éminemment individualisante, ou plus précisément individuante, une relation d'individuation - et ceci non pas en vertu de la psychologie personnelle de l'artiste, ou de sa volonté, ou encore de son passé scolaire, mais bien en raison de la nature même de l'art et du travail artistique ${ }^{7}$. Quand, par exemple, Anne Lopez, chorégraphe française en résidence dans un collège, émet à l'intention de tel enfant l'adresse suivante: Tu cherches en toi-même, comme le font souvent les artistes, il s'agit bien d'une adresse individuante, au centre de la dynamique relationnelle parce qu'elle est au cœur du travail artistique en tant que tel. Elle est éminemment au cœur du travail dramatique, et le propos relevé chez une chorégraphe pourrait l'être tout autant chez un metteur en scène. Ce que nous pouvons appeler l'institutionnalisation subjective est bien au cour du travail artistique comme travail éducatif, formateur. Cette considération confirme empiriquement la caractéristique majeure de l'expérience esthétique telle qu'elle peut être analysée sur le plan philosophique - une expérience indiscutablement sensorielle, émotionnelle et intellectuelle. Chaque enfant, chaque élève y est considéré, institué. L'expérience esthétique partagée, telle que l'intervention de l'artiste la permet, l'autorise, est bien alors une modalité exemplaire, prototypique, de la formation du sujet, d'une formation qui considère le sujet dans la pluralité et l'épaisseur de ses modalités d'existence, d'accomplissement de soi. On touche bien là à un enjeu éducatif plus que jamais capital.

\section{La Norme et la Loi}

Le champ de la normativité - c'est-à-dire de la capacité à produire et se donner des normes - recoupe un problème éducatif non moins crucial. Il est trop souvent réduit à un constat, discutable, de défaillance: la perte des normes, des repères, qu'il faudrait transmettre, le respect de la loi qu'il faudrait restaurer. Cette réduction enveloppe une conception trop étroitement verticale de la loi et de la norme en surplomb, qui ne peut suffire à éduquer à l'âge de l'individualisme 
démocratique, analysé par Marcel Gauchet (2002). La question éducative ne peut ignorer cette nouvelle donne et la nécessité de revisiter le champ de la normativité. Lart d'aujourd'hui, à sa façon, porte une même interrogation. Gérard Garouste, créateur de La Source ${ }^{8}$, interrogé au cours d'un entretien sur les raisons qui lui permettaient d'espérer que l'artiste puisse aider les élèves en difficulté, faisait cette réponse singulière:

Ce que l'artiste apporte d'abord? Une nécessaire et salutaire déstabilisation. Ce n'est pas l'absence de normes, mais la capacité à produire, travailler, déplacer la normativité qui importe... Ce qui manque le plus, ce qui a manqué le plus aux jeunes auxquels $L a$ Source a affaire: une présentation du monde. Un adulte là à côté pour montrer, désigner. Pour mettre la table dans un geste esthétique partagé ${ }^{9}$ (Kerlan, 2005, n.p.).

L'art commencerait donc là, à cette source-là, dans l'esthétique du quotidien, dans cette attention à la table bien mise; plus encore, l'estime de soi passerait par cette estime esthétique du monde. Et surtout: la portée éducative de l'art, il faudrait la rapporter à la normativité propre à la conduite esthétique.

Comme Daniel Sibony, Gérard Garouste démonte le lieu commun qui fait de l'art en éducation une donation de sens et de normes, de valeurs, dans un monde qui en serait dépourvu, un recours stabilisateur pour un monde instable. A l'inverse, il s'agit de refuser la fermeture du monde sur un sens donné, un sens tout fait, bouclé, sur un monde sans fenêtre.

Mais ce n'est pas refus de la norme et de la loi. Il s'agit d'une conception de la norme en résonance avec le sujet contemporain, lequel ne peut plus exister sous une unique norme en surplomb. En résonance donc avec les exigences de la démocratie contemporaine. On l'oublie trop souvent: le travail artistique est nécessairement confronté à la norme et à la loi, celles-là même de l'œuvre, celles-là même sans lesquelles l'œuvre ne pourrait advenir, et que l'œuvre elle-même produit, déplace, relance. L’expérience théâtrale est éminemment une expérience de cet ordre.

\section{Créativité, Création, Origine}

Le dernier thème, le thème de la créativité, recoupe de façon synthétique les deux thèmes précédents, celui du sujet et celui de la 
norme. Pas de travail créateur qui n'ait affaire aux normes, pas de travail créateur sur l'œuvre qui ne soit aussi un travail sur soi, qui ne soit travail de subjectivation. Le terme de créativité a fait l'objet de tant d'abus qu'on ose à peine l'utiliser. Et l'on est sans doute fondé à manifester sa réticence face à l'injonction de création et de créativité, ou au slogan tous créateurs. Pourtant, si l'on veut bien, avec Winnicott, «[...] envisager la créativité dans son acceptation la plus large, sans l'enfermer dans les limites d'une création réussie et reconnue» (1975, p. 91), on pourra se faire une idée du bénéfice éducatif, pour l'enfant, de l'expérience dans laquelle le fait entrer l'artiste. Winnicott invite à considérer la créativité «[...] comme la coloration de toute une attitude face à la réalité extérieure»: il s'agit avant tout, écrit-il, d'un:

[...] mode créatif de perception qui donne à l'individu le sentiment que la vie vaut la peine d'être vécue; ce qui s'oppose à un tel mode de perception, c'est une relation de complaisance soumise envers la réalité extérieure: le monde et tous ses éléments sont alors reconnus mais seulement comme étant ce à quoi il faut s'ajuster et s'adapter (Winnicott, 1975, p. 91).

La thématique de la créativité a donc bien à voir avec la fabrique du sujet contemporain, du sujet authentique, capable d'être pleinement lui-même, de se créer lui-même, avec les autres, de faire ouvre de soi avec les autres. Elle a bien affaire avec la démocratie, qui ne saurait s'accommoder, moins que jamais, de sujets préfabriqués, de sujets soumis, seulement ajustés et adaptés.

\section{Conclusion: l'experience esthetique, une source democratique necessaire}

Alors des artistes à l'école, pour quoi faire? A cette interrogation, la réponse pourrait tenir en une seule phrase: afin de permettre à tous les enfants d'entrer par l'expérience personnelle de l'art dans la conduite esthétique, de vivre une authentique expérience esthétique. Afin que la conduite esthétique enfantine soit accueillie dans la démarche de l'artiste, autorisée et promue dans le sillage de l'artiste et de son travail. Souvenons-nous de l'avertissement de Schiller: pour résoudre le problème politique, c'est-à-dire le problème de la liberté, c'est-à-dire le problème même de la démocratie, le chemin passe par l'esthétique. Parce que l'expérience esthétique à la source de l'art vaut par elle-même et en tant que telle, comme relation fondamentale au 
monde, comme modalité première d'être au monde. Oui, comme le pensait John Dewey, le rétablissement de la continuité entre l'expérience ordinaire et l'expérience esthétique est un véritable enjeu démocratique. On voudrait bien sûr que l'éducation artistique servent les apprentissages. Mais si l'expérience esthétique qui en est le cour peut ouvrir à l'enfant les chemins de la lecture et de l'instruction, comme le veut à bon droit l'école, c'est d'abord en lui ouvrant le monde dans sa lisibilité première. Le pédagogue Paolo Freire le disait merveilleusement: d'abord lire le monde, ensuite lire les mots. A l'ère du numérique, nous ferions bien de nous en souvenir.

On avancera, dans le prolongement de cette réflexion, une interrogation qu'on jugera peut-être culturellement iconoclaste: et si la véritable clé de la démocratisation était moins le taux de fréquentation des musées, des salles de concert et de théatre, des équipements numériques, que l'accès à l'expérience esthétique, à une vraie expérience esthétique? Et si cette troisième phase de la démocratisation était nécessaire à l'accomplissement des deux autres? Le théâtre, qui a l'âge de la démocratie telle que la Grèce sut l'inventer, et qui fut au cœur de l'œuvre et de la pensée de Schiller ${ }^{10}$, est sans doute de tous les arts l'un de ceux qui permet le mieux de le comprendre. Aujourd'hui, en France, et dans nombre d'autres pays, de plus en plus nombreux sont les élèves qui fréquentent les salles de spectacle, grâce à l'école qui les y conduit et aux gens de théâtre qui les accueillent. Et bien sûr il faut s'en réjouir. Mais aussi, certains soirs où le public scolaire est en nombre dans la salle de théâtre ou d'opéra, il arrive qu'on s'interroge: suffit-il d'être au théâtre, d'être allé au théâtre, pour que le théâtre, dans sa pleine dimension existe? Bien sûr non, quelque chose manque encore. La pratique théâtrale? Sans doute est-ce une expérience qui vous met mieux à l'unisson de la scène; mais, outre qu'il serait illusoire d'exiger de tous l'expérience du jeu théâtral, l'assemblée des spectateurs sans laquelle le théâtre n'existe pas encore diffère singulièrement d'une assemblée d'amateurs. Le partage de l'expérience théâtrale comme expérience esthétique est bien autre chose. Qu'on me permette de l'illustrer par un souvenir personnel et qui demeure pour moi exemplaire. La réputation d'Ariane Mouchkine et de son Théâtre du Soleil est telle bien au-delà des frontières que ce souvenir trouvera sans doute un écho chez plusieurs. Il s'agit d'une représentation de la pièce d'Hélène Cixious, Tambours sur la Digue, donnée à Lyon, dans un site architectural appelé Les Subsistances. Le spectacle 
était donné en plein air, il faisait assez frais, et selon sa tradition, le Théâtre du Soleil proposait à chaque spectateur de s'équiper d'une couverture protectrice. Et voilà l'image que je porte encore en moi, ancrée au plus fort de la représentation, dans un moment où seul le mot de communion convenait pour exprimer ce que vivait ensemble chacun des spectateurs: des centaines de têtes, mais un seul corps couvert de la même laine kaki. Voilà le miracle, la puissance du théâtre: unir le temps du spectacle dans une même expérience indissociablement sensorielle et intellectuelle, émotionnelle et réfléchie, ceux qui n'étaient que côte à côte. L'expérience du théâtre est bien une expérience tout à la fois esthétique et politique. Mieux encore: politique parce que esthétique. La question n'est pas: comment faire venir le peuple au théâtre? Elle est: comment l'expérience proposée par le théâtre peut-elle faire peuple, à quelles conditions? Comme l'on bien compris les hommes et les femmes de théâtre attachés à lui donner sa pleine dimension, le peuple, au sens pleinement politique du terme, qu'on ne confondra pas avec la foule, et encore moins avec les consommateurs, et même le public, n'est jamais déjà donné, il se produit, il advient comme événement, et le théâtre est l'un ses vecteurs d'avènement.

Donnons alors le dernier mot à l'une des lectrices les plus avisées de l'œuvre de Dewey: Joëlle Zask. Lectrice et interprète de Dewey, donc, Joëlle Zask pose la question: qu'est-ce que démocratiser? Méditons sa réponse.

"Démocratiser ne signifie pas mettre un même bien à la disposition d'un nombre croissant de gens» (Zask, 2003, p. 68). Pour notre part, nuançons ou précisons: démocratiser ne signifie pas seulement mettre un même bien à la disposition du plus grand nombre. Qu'estce que cela singifie donc de plus? Joëlle Zask poursuit ainsi:

Cela signifie veiller à la distribution sociale des biens de sorte que l'individualité de chacun soit respectée et encouragée. Une société démocratique est une société dans laquelle chaque personne peut bénéficier des ressources qui progressivement la constituent comme personne, de la naissance à la mort, et même dans la mémoire de ceux qui lui survivent. Or c'est précisément cette conviction qui est celle que les enseignements artistiques ont fait leur [...] (Zask, 2003, p. 68). 


\section{Notes}

${ }^{1}$ Dans sa forme originelle, cet article était une conférence donnée à Paris dans le cadre d'un colloque tenu au Sénat le 23 juin 2014, en présence de Madame la ministre de la Culture et de Monsieur le ministre de l'Éducation. Le collectif Pour l'Éducation, par l'Art, en collaboration avec Madame la sénatrice Marie-Christine Blandin qui l'accueillait, avait pris l'initiative de ce colloque avec l'ambition d'alerter les responsables politiques sur l'importance et les enjeux de l'éducation artistique. Ce propos s'adressait au monde politique français. Le propos a été repris, afin de lui donner les développements et les éclaircissements nécessaires.

${ }^{2}$ Les Lettres sont ici citées dans l'édition bilingue allemand-français de 1943 (traduction Robert Leroux) et reprise en 1992 aux éditions Aubier.

${ }^{3}$ Cf. Evaluer les Effets des Pratiques Artistiques et Culturelles: symposium européen et international de recherche (2008).

${ }^{4}$ Le site de ce dispositif permet d'en suivre les objectifs et les activités: <http://www.eal. lyon.fr/enfance/>.

${ }^{5}$ Ces deux dispositifs ont fait l'objet d'un suivi et d'une étude scientifiques: Kerlan (2005) et Carraud et al. (2015).

${ }^{6}$ Pour un développement plus conséquent, voir Kerlan (2011).

${ }^{7}$ Sur ce thème, voir Zask (2003).

${ }^{8}$ La Source est un lieu éducatif créé par le peintre Gérard Garouste, et géré dans le cadre d'une association. Ce lieu est implanté dans un petit village de la Normandie française, et reçoit des élèves en difficulté au sein d'ateliers conduits par des artistes en résidence.

${ }^{9}$ Entretien repris sous le titre L'Art à la Source, aujourd'hui Encore et Toujours dans la revue en ligne Sens Public, et accessible sur son site: <http://www.sens-public.org/spip. php?article973>.

${ }^{10}$ Rappelons que Schiller fut avec Goethe, à Weimar, dès 1791, directeur du tout nouveau Théâtre de la Cour grand-ducale, devenu Théâtre National Allemand en 1918.

\section{Références}

BOLTANSKI, Luc; CHIAPELLO, Eve. Le Nouvel Esprit du Capitalisme. Paris: Gallimard, 1999.

DEWEY, John. L'Art comme Expérience. Pau: Éditions Farago, 2005.

DEWEY, John. Democracy and Education: an introduction to the philosophy of education. New York: Macmillan, 1916.

DUMONT, Fernand. Le Lieu de l'Homme: la culture comme distance et mémoire. Montréal: Bibliothèque Québécoise, 1994 [1968]. 
EVALUER LES EFFETS DES PRATIQUES ARTISTIQUES ET CULTURELLES: symposium européen et international de recherche [Evaluating the Impact of Arts and Cultural Education: a European and international symposium], 2007, Paris. Actes... Paris: Centre Pompidou/La Documentation Française, 2008. Édité en français et em anglais.

GAUCHET, Marcel. La Démocratie contre Elle-Même. Paris: Gallimard, 2002.

KERLAN, Alain. L'Art à la Source, aujourd'hui Encore et Toujours: deux entretiens avec Elisabeth et Gérard Garouste. Revue Sens Public, Paris, 01 juin 2012. Disponible sur: <http://www.sens-public.org/spip.php?article973>. Consulté le: 05 nov. 2014.

KERLAN, Alain. L’Art pour Éduquer? La Tentation Esthétique: contribution philosophique à l'analyse d'un paradigme. Québec: Presses de l'Université Laval, 2004.

KERLAN, Alain. L'Atelier de l'Artiste, Laboratoire Démocratique d'une Nouvelle Normativité? Revue Sens Public, 06 déc. 2011. Disponible sur: <http://www.sens-public.org/ spip.php?article894>. Consulté le: 05 nov. 2014.

KERLAN, Alain (Dir.). Des Artistes à la Maternelle. Lyon/Paris: Scéren/CNDP, 2005.

KERLAN, Alain et al. (Dir.). Un Collège Saisi par les Arts. Paris: Éditions de l'Attribut, 2015. À paraître.

MARCUSE, Herbert. The Aesthetic Dimension. Boston: Beacon Press, 1978.

RANCIÈRE, Jacques. La Nuit des Prolétaires: archives du rêve ouvrier. Paris: Fayard, 1981.

RANCIÈRE, Jacques. Le Partage du Sensible. Paris: La Fabrique, 2000.

READ, Herbert. The Education of Free Men. London: Freedom Press, 1944.

READ, Herbert. Education through Art. London: Faber and Faber, 1967.

ROUSSEAU, Jean-Jacques. Émile, ou de l'Éducation. Paris: Gallimard, 1969. (Collection Euvres Complètes.)

ROUSSEAU, Jean-Jacques. Les Rêveries du Promeneur Solitaire. Paris: Gallimard, 1959. (Collection Euvres Complètes.)

SCHAEFFER, Jean-Marie. Adieu à l'Esthétique. Paris: PUF, 2000.

SCHILLER, Friedrich von. Lettres sur l'Éducation Esthétique de l'Homme. Traduction: Leroux Robert. Paris: Aubier, 1992 [1943].

SIBONY, Daniel. Création: essai sur l'art contemporain. Paris: Seuil, 2005.

TAYLOR, Charles. Le Malaise de la Modernité. Paris: Cerf, 2002.

WINNICOTT, Donald. Jeu et Réalité: l'espace potentiel. Paris: Gallimard, 1975 [1971]. ZASK, Joëlle. Art et Démocratie: les peuples de l'art. Paris: PUF, 2003. 
E-ISSN 2237-2660

Alain Kerlan est philosophe, professeur des universités en poste à l'Université Lumière Lyon 2, où il exerce les fonctions de Directeur de l'Institut des Sciences et des Pratiques d'Éducation et de Formation (ISPEF). Il est l'auteur de nombreux ouvrages. E-mail: alain.kerlan@univ-lyon2.fr

Ce texte inédit, révisé par Annelyse Gayraud, est également publié en portugais dans ce numéro.

Reçu le 29 juillet 2014 Accepté le 05 novembre 2014 